\title{
EFECTO DEL CATIÓN, DEL ANIÓN Y DEL CO-IÓN SOBRE LA AGREGACIÓN DE LÍQUIDOS IÓNICOS EN SOLUCIÓN ACUOSA
}

\author{
Nólides Marina Guzmán, José Francisco Fernández*, Mónica Parada, Carlos Orbegozo, María Alejandra Rodríguez \\ y Alida Padrón \\ Escuela de Ingeniería Química, Universidad Central de Venezuela, 1040 Caracas, Venezuela \\ Jorg Thöming \\ Zentrum für Umweltforschung und nachhaltige Technologien, Universität Bremen, 28359 Bremen, Deutschland
}

Recebido em 10/12/09; aceito em 31/5/10; publicado na web em 1/9/10

\begin{abstract}
CATION, ANION AND CO-ION EFFECT ON THE AGGREGATION OF IONIC LIQUIDS IN AQUEOUS SOLUTION. The aggregation behavior of thirteen 1-alkyl-3-methylimidazolium based ionic liquids in aqueous solution is presented, considering variations of the alkyl side chain length as well as the anionic moiety. Cation and anion molecular volumes are selected as appropriate molecular descriptors. Additionally, the existing relationship between critical micelle concentration (CMC) and electrolyte concentration in solution is established, aiming to clarify ion effects. CMC values were obtained by measuring electrical conductivity and surface tension. It was confirmed that aggregation of ionic liquids in aqueous solution and in presence of inorganic salts is affected by the factors developed in this study.
\end{abstract}

Keywords: ionic liquids; aggregation; critical micelle concentration.

\section{INTRODUCCIÓN}

Los líquidos iónicos (LIs) de la familia 1-alquil-3-metil-imidazolio muestran un comportamiento similar a otros compuestos anfifílicos en solución acuosa. Este comportamiento se debe a la presencia de una cadena alquílica generalmente larga (hidrofóbica) y un grupo polar con carga deslocalizada (hidrofílico). En los últimos años muchos investigadores han reportado en sus estudios valores de la Concentración Micelar Crítica (CMC) para diversos líquidos iónicos de ésta y otras familias, como lo reportan Luczak et al.. ${ }^{1}$

La CMC representa la mínima concentración requerida para que la agregación de un compuesto anfifílico determinado suceda. Tradicionalmente, se determina a partir del cambio de tendencia observado para diferentes propiedades como una función de la concentración, entre ellas tensión superficial y conductividad eléctrica. Así mismo, la CMC depende de diversos factores, tales como la estructura del líquido iónico, la forma en que se constituyen los agregados y las características del medio en el cual se solubiliza el líquido iónico. ${ }^{2}$ En este sentido, la temperatura y la presencia de sales afectan el proceso de agregación.

La relación más conocida entre la CMC y la estructura química de los surfactantes es la ecuación de Stauff-Klevens, aplicable solamente a series homólogas. ${ }^{3}$ Dicha ecuación indica que existe una relación logarítmica entre la CMC y el número de átomos de carbono de la cadena alquílica:

$$
\log C M C=A+B \cdot n
$$

donde $A$ y $B$ son constantes para una serie homóloga particular y una temperatura dada, y $n$ es el número de átomos de carbono de la cadena hidrocarbonada. Sin embargo, no existe hasta la fecha ninguna investigación que haya estudiado de forma sistemática el efecto del anión sobre la CMC. ${ }^{1}$

De esta manera, la primera parte de este estudio intentará contribuir a llenar este vacío de información, mediante el estudio de la agre-

*e-mail: jo_fe@uni-bremen.de gación de líquidos iónicos de la familia 1-alquil-3-metil-imidazolio en solución acuosa, considerando las variaciones tanto del catión en términos de la longitud de la cadena alquílica como del anión. Para ello se seleccionaron los volúmenes moleculares del catión y del anión como descriptores moleculares apropiados. ${ }^{4}$

Por otra parte, la adición de electrolitos altera el ambiente físicoquímico de la solución y, en consecuencia, afecta el valor de la CMC. Los términos co-ión y contra-ión adquieren especial importancia en este caso. Una micela es una partícula coloidal cargada eléctricamente, la cual está rodeada por iones disociados de carga eléctrica de signo contrario y que se denominan contra-iones. Si al medio en el que se encuentran las micelas y los contra-iones se le añade un electrolito, el mismo proveerá contra-iones adicionales y también co-iones, los cuales tienen una carga eléctrica del mismo signo que la carga eléctrica de la micela. ${ }^{5}$

Así mismo, los iones pequeños con alta densidad de carga (cosmótropos) se hidratan fácilmente en solución y por lo tanto interactúan de manera desfavorable con otros solutos hidrofóbicos. Por el contrario, los iones grandes con baja densidad de carga (caótropos) se asocian favorablemente con otros solutos hidrofóbicos. De esta manera, es posible establecer que la estructura de la sal tiene un efecto importante sobre el proceso de agregación, así como también sobre la solubilidad del líquido iónico en solución.

Estas ideas sugieren que los iones cosmotrópicos (bien hidratados), como el $\mathrm{Mg}^{+2}$, se excluyen a sí mismos del contacto con los solutos hidrofóbicos, disminuyendo la solubilidad de dichos solutos. Este proceso se conoce como salting-out. Por otra parte, los iones caótropos, como el $\mathrm{K}^{+}$, estabilizan la interfase soluto-agua asociándose preferencialmente con el soluto, lo cual conduce a un aumento en la solubilidad del soluto hidrofóbico, mejor conocido como salting-in. ${ }^{6}$

A pesar de que la información asociada al proceso de agregación de líquidos iónicos en solución acuosa es relativamente abundante, es poco lo que se sabe acerca del comportamiento de dichos compuestos en presencia de sales inorgánicas. En tal sentido, en este estudio no sólo se determinará el efecto de la naturaleza de la sal empleada sobre el proceso de agregación, relacionada con la cosmotropicidad o caotropicidad de los co-iones seleccionados, sino que además se 
establecerá la relación existente entre la CMC y la concentración del electrolito, tratando de esclarecer el efecto que tienen tanto el co-ión como el contra-ión. Ambos efectos ayudarán a comprender mejor los procesos de agregación que se llevan a cabo en medios acuosos salinos, dada la importancia de estos últimos en los procesos biológicos y ambientales.

\section{PARTE EXPERIMENTAL}

\section{Materiales}

Los líquidos iónicos empleados fueron producidos por Merck KGaA (Darmstatd, Alemania), todos con una pureza $\geq 98,0 \%$. En la Tabla 1 se presentan los líquidos iónicos empleados y sus características, así como la nomenclatura usada en este artículo. Se empleó adicionalmente cloruro de potasio (J.T. Baker, pureza $\geq 99,5 \%$ ) y cloruro de magnesio hexahidratado (J.T. Baker, pureza $\geq 99,5 \%$ ). Para preparar las soluciones correspondientes se utilizó agua ultra pura de baja conductividad (Direct Q-3 UV System, Millipore).

Tabla 1. Líquidos iónicos empleados

\begin{tabular}{|c|c|c|}
\hline Nombre químico según IUPAC & $\begin{array}{l}\text { Peso } \\
\text { molecular }\end{array}$ & $\begin{array}{l}\text { Nomenclatura } \\
\text { empleada }\end{array}$ \\
\hline Cloruro de 1-hexil-3-metil-imidazolio & 202,73 & {$[\mathrm{HMIM}][\mathrm{Cl}]$} \\
\hline $\begin{array}{l}\text { Tetrafluoroborato de 1-hexil-3-metil-imidaz- } \\
\text { olio }\end{array}$ & 254,08 & {$[\mathrm{HMIM}]\left[\mathrm{BF}_{4}\right]$} \\
\hline $\begin{array}{l}\text { Hexafluorofosfato de 1-hexil-3-metil-imid- } \\
\text { azolio }\end{array}$ & 312,24 & {$[\mathrm{HMIM}]\left[\mathrm{PF}_{6}\right]$} \\
\hline $\begin{array}{l}\text { Bis(trifluorometil-sulfonilo)imiduro de } \\
\text { 1-hexil-3-metil-imidazolio }\end{array}$ & 447,41 & {$[\mathrm{HMIM}]\left[\mathrm{NTf}_{2}\right]$} \\
\hline Cloruro de 1-octil-3-metil-imidazolio & 230,78 & {$[\mathrm{OMIM}][\mathrm{Cl}]$} \\
\hline $\begin{array}{l}\text { Tetrafluoroborato de 1-octil-3-metil-imidaz- } \\
\text { olio }\end{array}$ & 282,13 & {$[\mathrm{OMIM}]\left[\mathrm{BF}_{4}\right]$} \\
\hline $\begin{array}{l}\text { Hexafluorofosfato de 1-octil-3-metil-imidaz- } \\
\text { olio }\end{array}$ & 340,29 & {$[\mathrm{OMIM}]\left[\mathrm{PF}_{6}\right]$} \\
\hline $\begin{array}{l}\text { Bis(trifluorometil-sulfonilo)imiduro de 1-octil- } \\
\text { 3-metil-imidazolio }\end{array}$ & 475,46 & {$[\mathrm{OMIM}]\left[\mathrm{NTf}_{2}\right]$} \\
\hline Cloruro de 1-decil-3-metil-imidazolio & 258,83 & [DMIM] $[\mathrm{Cl}]$ \\
\hline $\begin{array}{l}\text { Tetrafluoroborato de 1-decil-3-metil-imidaz- } \\
\text { olio }\end{array}$ & 310,18 & {$[\mathrm{DMIM}]\left[\mathrm{BF}_{4}\right]$} \\
\hline Cloruro de 1-dodecil-3-metil-imidazolio & 286,89 & [DDMIM] $[\mathrm{Cl}]$ \\
\hline Cloruro de 1-hexadecil-3-metil-imidazolio & 343,00 & {$[\mathrm{HDMIM}][\mathrm{Cl}]$} \\
\hline Cloruro de 1-octadecil-3-metil-imidazolio & 371,05 & {$[\mathrm{ODMIM}][\mathrm{Cl}]$} \\
\hline
\end{tabular}

\section{Métodos}

Las muestras fueron preparadas en un amplio rango de concentraciones, empleando los compuestos originales o soluciones concentradas de los mismos. Para ello se preparó una solución madre con la concentración de sal deseada, la cual se empleó en sustitución del agua ultra pura para la preparación de las soluciones correspondientes para el estudio con sales inorgánicas. Todas las soluciones se prepararon en recipientes de vidrio y las mediciones se llevaron a cabo un día después de la preparación para asegurar que el proceso de agregación alcanzó una condición de equilibrio.

Las mediciones de conductividad fueron realizadas con dos equipos: Oakton(R) CON 510 Benchtop Meter (Fisher Scientific) y Orion 3-Star Plus Benchtop Meter (Thermo Scientific), con sus respectivos electrodos. Cada electrodo fue calibrado usando diferentes estándares de cloruro de potasio, antes y después de cada serie de mediciones.
Por otra parte, las mediciones de tensión superficial se realizaron en un tensiómetro de anillo de Du Noüy (Fisher Scientific). El equipo fue calibrado regularmente empleando un set de pesas marca Ohaus. La temperatura se mantuvo constante e igual a $25 \pm 0,2{ }^{\circ} \mathrm{C}$ para todas las mediciones, empleando un baño térmico (Digisystem, DSB-1000D).

La conductividad y la tensión superficial de cada muestra se midieron por triplicado y se reportó el valor medio junto con su desviación. Posteriormente, a las mediciones de conductividad de la solución $(k)$ se les sustrajo la conductividad correspondiente al solvente $\left(k_{0}\right)$, de manera de obtener la conductividad de la sustancia en estudio $(\Delta k)$, la cual fue graficada en función de la concentración $(C)$ de dicha sustancia en la solución correspondiente. El método empleado para determinar la CMC está bien documentado en la literatura. ${ }^{7}$

Finalmente, los volúmenes moleculares se calcularon por medio del programa MOPAC2007 (Molecular Orbital PACkage) y el método semiempírico PM6 para optimización geométrica. ${ }^{8}$ El efecto del solvente rodeando la molécula fue considerado empleando el modelo COSMO (COnductor-like Screening MOdel), con una constante dieléctrica equivalente a la del agua a $20^{\circ} \mathrm{C}(80,1)$.

\section{RESULTADOS Y DISCUSIÓN}

\section{Efecto de la longitud de la cadena alquílica}

Para determinar el efecto de la longitud de la cadena carbonada sobre el proceso de agregación, se emplearon líquidos iónicos del tipo cloruro de 1-alquil-3-metil-imidazolio, con cadenas carbonadas entre 6 y 18 átomos de carbono.

Empleando mediciones de conductividad eléctrica fue posible determinar la CMC para estos compuestos, cuyos valores se presentan en la Tabla 2. A medida que aumenta la longitud de la cadena carbonada se observa una disminución de la CMC, debido a que el componente hidrofóbico del catión se hace cada vez más importante y se necesita una menor concentración del compuesto para que se dé la formación de micelas. Para el caso de una cadena carbonada de 6 átomos de carbono no se detectó agregación.

Tabla 2. Efecto del catión sobre la CMC para líquidos iónicos de la familia cloruro de 1-alquil-3-metil-imidazolio

\begin{tabular}{lc}
\hline Líquido iónico & Concentración micelar crítica, CMC $\left(\mathrm{mol} / \mathrm{m}^{3}\right)$ \\
\hline$[\mathrm{HMIM}][\mathrm{Cl}]$ & No detectable \\
{$[\mathrm{OMIM}][\mathrm{Cl}]$} & $179 \pm 24$ \\
{$[\mathrm{DMIM}][\mathrm{Cl}]$} & $48,7 \pm 6,1$ \\
{$[\mathrm{DDMIM}][\mathrm{Cl}]$} & $13,4 \pm 1,2$ \\
{$[\mathrm{HDMIM}][\mathrm{Cl}]$} & $1,4 \pm 0,4$ \\
{$[\mathrm{ODMIM}][\mathrm{Cl}]$} & $0,37 \pm 0,15$ \\
\hline
\end{tabular}

Si se grafican estos valores de CMC en función del número de átomos de carbono de la cadena alquílica, en escala semilogarítmica se obtiene una línea recta. De acuerdo a la ecuación empírica de Stauff-Kleven, el coeficiente $A$ (de la ecuación 1) es prácticamente igual a $-\log 2(-0,301)$, lo cual indica que la adición de un grupo metileno $\left(-\mathrm{CH}_{2}-\right)$ a la cadena carbonada disminuye aproximadamente la CMC en un factor igual a $2 .{ }^{9}$

Con el fin de relacionar la CMC con el volumen molecular del catión del líquido iónico, se calcularon por medio del programa MOPAC2007 los volúmenes correspondientes a los diferentes cationes $\left(V^{+}\right)$y dichos valores se graficaron en función del número de carbonos de la cadena alquílica, obteniéndose una buena correlación $\left(\mathrm{R}^{2}=0,99982\right)$. 
En consecuencia, es posible transformar la ecuación empírica de Stauff-Klevens, usando logaritmo neperiano en lugar del logaritmo decimal, de la siguiente manera:

$$
\operatorname{LnCMC}=-0,0283 \cdot V^{+}+13,52
$$

A partir de los datos de la literatura para diversos líquidos iónicos de la familia 1-alquil-3-metil-imidazolio con dos aniones diferentes, cloruro y bromuro, se observa en la Figura 1 un comportamiento similar al presentado al graficar la CMC como función del número de átomos de carbono de la cadena carbonada.

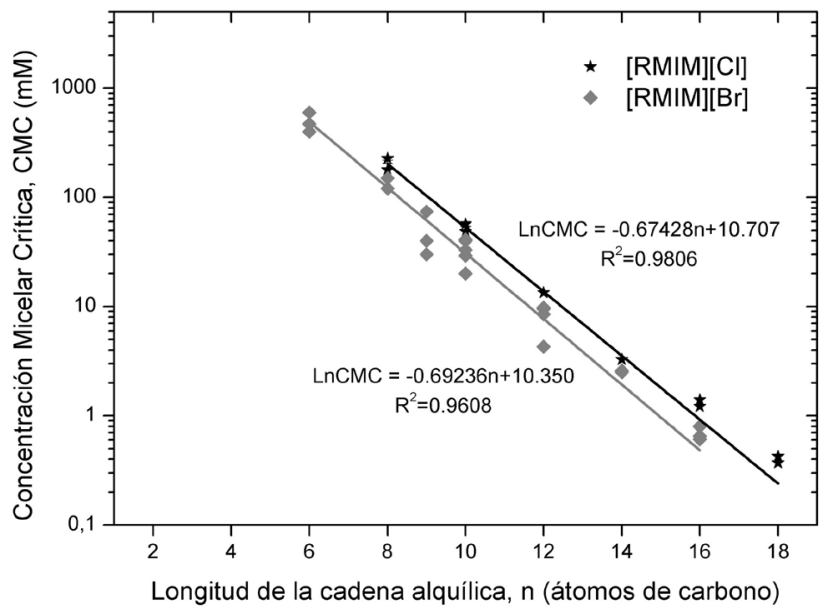

Figura 1. Dependencia de la CMC con la longitud de la cadena alquílica para líquidos iónicos del tipo 1-alquil-3-metil-imidazolio con diversos aniones

Empleando la relación entre el volumen del catión y la longitud de la cadena alquílica, es posible expresar las ecuaciones reportadas en la Figura 1 en forma análoga a la Ecuación 2:

$$
\begin{aligned}
& \text { Anión cloruro: } \operatorname{LnCMC}=-0,0294 \cdot V^{+}+13,96 \\
& \text { Anión bromuro: } \operatorname{LnCMC}=-0,0301 \cdot V^{+}+13,70
\end{aligned}
$$

Sin perder de vista los errores asociados a la determinación de la CMC (técnicas y/o métodos), es posible mediante la comparación de las ecuaciones anteriores, establecer lo siguiente: en el caso de las Ecuaciones 2 y 3, se observa una buena similitud entre los coeficientes de ambas expresiones, considerando que los primeros provienen de las mediciones experimentales realizadas en este estudio y los segundos son producto de mediciones experimentales realizadas por otros investigadores. En el caso de las Ecuaciones 3 y 4, se observa que el coeficiente asociado al volumen molecular del catión $\left(V^{+}\right)$es prácticamente el mismo, lo cual es de esperarse dado que los líquidos iónicos sólo difieren entre sí en el anión empleado.

De esta manera, se confirma la hipótesis acerca del uso de los volúmenes moleculares como descriptores adecuados para la predicción de la CMC.

\section{Efecto del anión}

Una de las indicaciones más resaltantes de la influencia del anión sobre las propiedades físicas de los líquidos iónicos, se evidencia en la solubilidad en agua. ${ }^{10}$ Por ello, se decidió estudiar el efecto que sobre la agregación de los líquidos iónicos en solución acuosa tienen los aniones mostrados en la Figura 1.
En tal sentido, se realizaron mediciones de tensión superficial para diferentes soluciones de líquidos iónicos, variando la concentración desde cero (agua pura) hasta un valor cercano a la solubilidad del líquido iónico en agua a la temperatura de trabajo $\left(25 \pm 0,2{ }^{\circ} \mathrm{C}\right)$.

Los resultados indican que el nuevo anión (tetrafluoroborato, $\mathrm{BF}_{4}^{-}$) inhibe la formación de agregados para aquellos cationes que en el caso del anión cloruro presentaron agregación: [OMIM] y [DMIM]. De esta manera, es posible pensar que existe un efecto estructural asociado al cambio en el anión. Para el caso del [HMIM] se combinan dos efectos que desfavorecen la agregación: cadena hidrocarbonada corta y anión de mayor tamaño.

Siguiendo estos razonamientos, los resultados experimentales ratifican el efecto estructural que tiene el anión sobre el proceso de agregación, ya que al emplear un anión cada vez más grande se reduce la tensión superficial, pero no se favorece la formación de agregados. En la Tabla 3 se presentan los volúmenes moleculares correspondientes a los aniones empleados en este estudio, calculados con el programa MOPAC2007.

Tabla 3. Volumen molecular de los aniones empleados

\begin{tabular}{cc}
\hline Anión & Volumen molecular $\left(\AA^{3}\right)$ \\
\hline$[\mathrm{Cl}]$ & 36,09 \\
{$\left[\mathrm{BF}_{4}\right]$} & 71,13 \\
{$\left[\mathrm{PF}_{6}\right]$} & 98,32 \\
{$\left[\mathrm{NTf}_{2}\right]$} & 222,36 \\
\hline
\end{tabular}

Evidencia adicional a favor de este argumento se desprende de la Figura 1, donde es posible observar agregación para algunos de los compuestos empleados en este estudio, pero con el anión bromuro. Si se comparan los valores correspondientes a los volúmenes moleculares de los aniones cloruro $\left(36,09 \AA^{3}\right)$ y bromuro $\left(42,21 \AA^{3}\right)$, se concluye que el incremento en el tamaño del anión no es lo suficientemente importante para inhibir el proceso de agregación, pero sí para que éste se produzca a una menor concentración. Este último fenómeno se explicaría en función de la mayor caotropicidad del anión bromuro. De esta manera, los aniones bromuros son más fácilmente adsorbidos sobre la superficie de los agregados, reduciendo las repulsiones electrostáticas entre los cationes y facilitando la agregación. ${ }^{11}$

Por último, a medida que se aumenta el tamaño del anión, se reduce la solubilidad del líquido iónico en agua de forma drástica, lo que induce a pensar que la formación de una segunda fase líquida de líquido iónico puro es una situación termodinámicamente más estable que la formación de agregados en solución. En este orden de ideas, Blesic et al. ${ }^{12}$ concluyen que el anión induce la separación de fases antes de que la agregación pueda ocurrir.

\section{Relación entre CMC y la estructura del líquido iónico}

Como se describió anteriormente, es posible establecer una relación entre CMC y el volumen molecular del catión $\left(V^{+}\right)$bajo ciertos niveles de confiabilidad. Lamentablemente, no existe aún suficiente información en la literatura para poder establecer de manera similar el efecto que el volumen molecular del anión $(V)$ tiene sobre la CMC.

Si se tiene en cuenta que un incremento en el tamaño del anión acarrea una disminución de la solubilidad en agua, se puede comprender por qué en el caso de los líquidos iónicos empleados en este estudio no fue posible identificar agregación alguna.

Estos resultados nos llevan a sugerir la posibilidad de crear un “mapa de agregación” para el caso de los líquidos iónicos de la familia 1-alquil-3-metil-imidazolio, tal como se presenta en la Figura 2. Dicho mapa contiene en los ejes $x$ e $y$ los volúmenes moleculares 


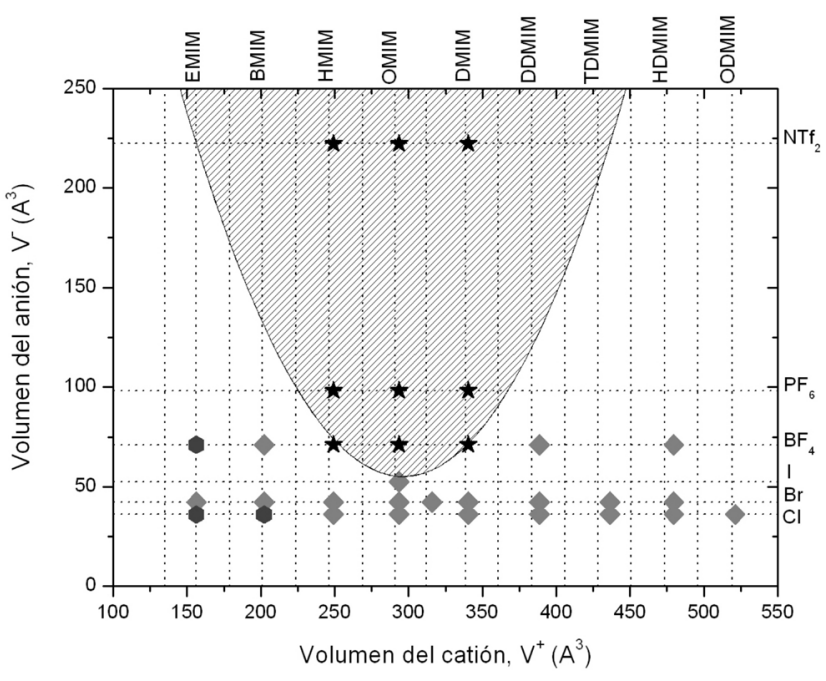

Figura 2. Mapa de agregación propuesto para líquidos iónicos de la familia 1-alquil-3-metil-imidazolio. La zona rayada indica la región donde no se produce agregación alguna. Los diversos símbolos (su significado se aclara en el texto) representan la presencia o no de agregación para una combinación catión-anión dada, de acuerdo a los resultados disponibles en la literatura especializada

del catión $\left(V^{+}\right)$y anión $\left(V^{*}\right)$, respectivamente. Finalmente, el eje $z$ (no mostrado en la Figura 2) correspondería a los valores de la CMC.

Los rombos indican aquellas combinaciones catión-anión para las cuales se tienen referencias de que la agregación ocurre y, por lo tanto, la CMC existe. Las estrellas, por el contrario, indican aquellas combinaciones catión-anión donde no ocurre agregación y en consecuencia, no aplica la definición de CMC.

Vale destacar que los puntos señalados como hexágonos se corresponden a valores de $\mathrm{CMC}$ reportados en la literatura que en este estudio no se consideran totalmente confiables, pues se corresponden a cationes de corta cadena alquílica para los cuales se reporta agregación a concentraciones altas. Este hecho requiere de investigación adicional, pues pareciera ser que la agregación se da como consecuencia de la cantidad elevada de moléculas en solución y no como consecuencia de la estructura del líquido iónico en sí.

La zona rayada (en este caso, delimitada por una frontera definida con fines ilustrativos y sin basamento teórico) indicaría entonces aquellas combinaciones para las cuales no se esperaría observar agregación alguna. De la Figura 2 se desprende que la agregación sería posible con aniones voluminosos, siempre y cuando la cadena alquílica sea lo suficientemente larga. Sin embargo, se debe tener en cuenta que cationes voluminosos y aniones voluminosos deben producir una baja solubilidad en agua, por lo que la separación de fases tendría prioridad sobre la agregación.

Puesto que el "mapa de agregación" es sólo una propuesta elaborada a partir de la evidencia experimental acumulada hasta este momento, se requiere aún de mucha investigación adicional para poder complementar la misma. Además, debería superponerse a este mapa el correspondiente a la solubilidad en fase acuosa, dado que los dos fenómenos parecieran competir entre sí para el caso de estructuras de gran tamaño.

\section{Efecto de la naturaleza de la sal}

$\mathrm{Al}$ analizar los resultados de las mediciones conductimétricas realizadas a $25 \pm 0,2{ }^{\circ} \mathrm{C}$, para una serie de soluciones de [DDMIM] [Cl] en una solución de $\mathrm{KCl}$ con una concentración $1 \mathrm{~mol} / \mathrm{m}^{3}$ y una solución de $\mathrm{MgCl}_{2}$ de la misma concentración, ambas comparadas con lo que sucede cuando el solvente es agua ultra pura, es posible apreciar un cambio de tendencia en el rango de concentraciones estudiado, lo cual permite concluir que este líquido iónico forma agregados también en soluciones que contienen sales inorgánicas disueltas, con la particularidad de que en ambos casos la CMC obtenida es mucho menor a la reportada en ausencia de electrolitos, como se resume en la Tabla 4.

Tabla 4. Efecto de la estructura de la sal sobre la CMC para líquidos iónicos de la familia cloruro de 1-alquil-3-metil-imidazolio

\begin{tabular}{lccc}
\hline $\begin{array}{l}\text { Líquido } \\
\text { iónico }\end{array}$ & \multicolumn{3}{c}{ Concentración micelar crítica, $\mathrm{CMC}\left(\mathrm{mol} / \mathrm{m}^{3}\right)$} \\
& $\begin{array}{c}\text { Agua } \\
\text { ultra pura }\end{array}$ & $\begin{array}{c}\text { Solución } \\
1 \mathrm{~mol} / \mathrm{m}^{3} \mathrm{de} \mathrm{KCl}\end{array}$ & $\begin{array}{c}\text { Solución } \\
1 \mathrm{~mol} / \mathrm{m}^{3} \mathrm{de} \mathrm{MgCl}_{2}\end{array}$ \\
\hline$[\mathrm{DDMIM}][\mathrm{Cl}]$ & $13,4 \pm 1,2$ & $12,1 \pm 0,2$ & $8,4 \pm 0,1$ \\
{$[\mathrm{ODMIM}][\mathrm{Cl}]$} & $0,37 \pm 0,15$ & $0,37 \pm 0,01$ & No detectable \\
\hline
\end{tabular}

Así mismo, en la Tabla 4 se presentan los resultados obtenidos para otro líquido iónico de la familia en estudio, el [ODMIM][Cl]. En el caso de la solución de $\mathrm{KCl}$ es difícil hablar de una reducción de la $\mathrm{CMC}$, dado que los valores obtenidos son similares, más sin embargo, el rango de concentraciones donde es posible encontrar la CMC en presencia de $\mathrm{KCl}$ está mucho mejor definido que en el caso del agua ultrapura.

No obstante, cuando se empleó la solución de $\mathrm{MgCl}_{2}$ no fue posible detectar un cambio de tendencia en el rango de concentraciones estudiado. Por el contrario, la solución obtenida era inestable a temperatura ambiente evidenciándose la formación de una segunda fase, dada la hidrofobicidad asociada a la cadena alquílica de 18 átomos de carbonos unida al anillo imidazolio.

Los resultados obtenidos para ambos líquidos iónicos pueden explicarse en función de la naturaleza del catión que conforma la sal inorgánica añadida a la solución (co-ión). El catión magnesio es de naturaleza cosmótropa, mientras que el catión potasio es de naturaleza caótropa. ${ }^{13}$

Los iones cosmótropos son solvatados con mucha más facilidad por el agua que los iones caótropos. Esta habilidad de los iones cosmótropos de formar enlaces intermoleculares, tipo puentes de hidrógeno, representa una modificación del ambiente físicoquímico de la solución. De esta forma, el electrolito no sólo ocupa un espacio físico en el sistema, sino que también empieza a competir por el agua libre con el líquido iónico.

Si la habilidad del catión de la sal para solvatarse es superior a la del catión del líquido iónico, disminuye la solubilidad de este último, impidiéndole que se solvate con el agua libre del sistema. De esta manera, el catión del líquido iónico se asociará con otros cationes de su misma especie favoreciendo la formación de agregados a menores concentraciones como en el caso del [DDMIM][Cl], o produciendo el fenómeno de salting-out, como en el caso del [ODMIM][Cl].

\section{Efecto de la concentración de la sal}

Para evaluar el efecto de la concentración de ambas sales sobre el proceso de agregación del [DDMIM][Cl] se escogieron tres concentraciones diferentes: 0,$1 ; 1$ y $10 \mathrm{~mol} / \mathrm{m}^{3}$. En la Figura 3 se observan los resultados obtenidos, apreciándose que el efecto de aumentar la concentración del electrolito es mucho más marcado para el caso del $\mathrm{MgCl}_{2}$. Este fenómeno es atribuido al aumento de la concentración del contra-ión (en este caso, el anión cloruro), lo cual disminuye la repulsión entre los cationes del líquido iónico y, por ende, la oposición a la formación de agregados se reduce. ${ }^{12}$

De esta manera, se decidió establecer una relación entre la CMC y la concentración total del anión cloruro de acuerdo al modelo desar- 

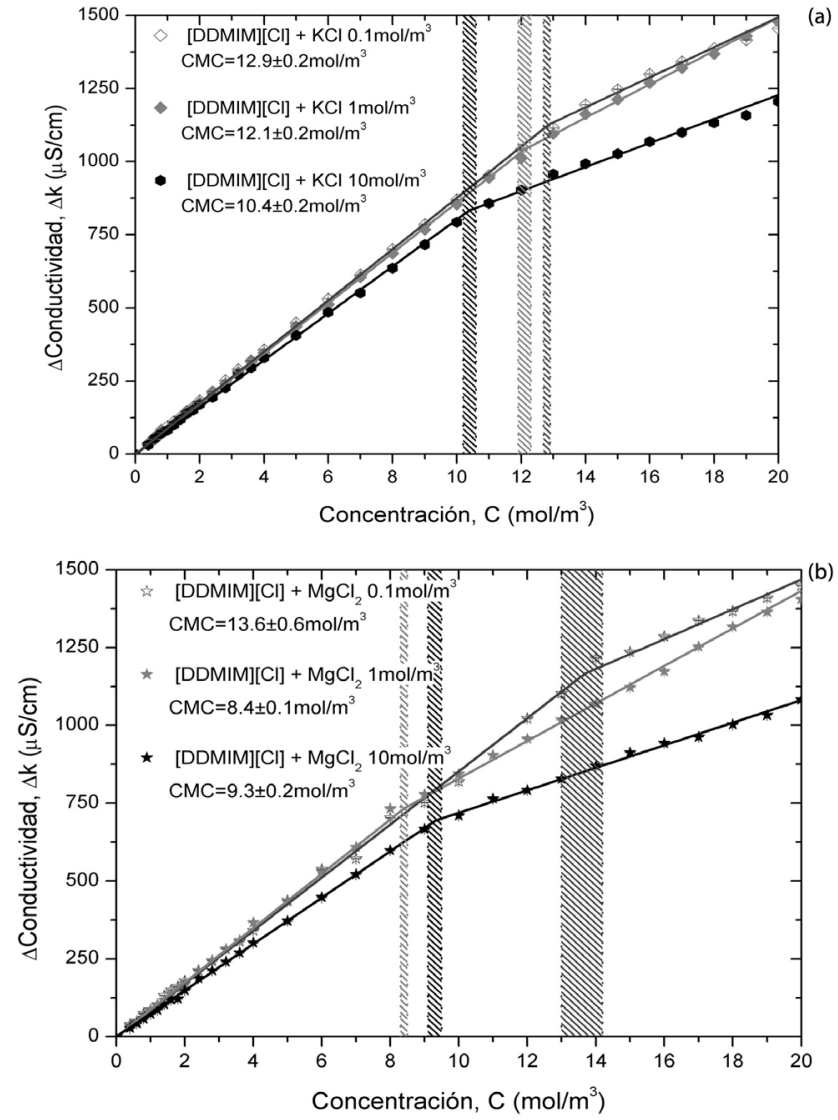

Figura 3. Efecto de la concentración de la sal sobre la agregación del cloruro de 1-dodecil-3-metil-imidazolio en presencia de: (a) $\mathrm{KCl}$ y (b) $\mathrm{MgCl}_{2}$

rollado por Corrin y Harkins. ${ }^{14}$ Estos autores indican que el logaritmo de la CMC es una función lineal del logaritmo de la concentración total del ión opuesto en carga a áquel que se agrega. Dicha función viene dada por la Ecuación 5:

$$
\log C M C=a+b \cdot \log C_{C l^{-}}
$$

donde $C_{C \text { 俑 }}$ es la concentración total de aniones cloruro (provenientes tanto del líquido iónico como de la sal añadida), y $a$ y $b$ son los parámetros a obtener mediante el ajuste.

Entre sus conclusiones Corrin y Harkins indican además que la concentración y la carga del ión que posee la misma carga que el que se asocia (co-ión, en este caso el catión potasio o el catión magnesio) pareciera no tener efecto alguno sobre la CMC. Sin embargo, los resultados obtenidos en la Figura 4 indican lo contrario.

El ajuste de los datos de CMC en presencia de una solución de $\mathrm{KCl}$ a este modelo es mucho mejor que el obtenido para los equivalentes en presencia de una solución de $\mathrm{MgCl}_{2}$; sin embargo, ambos parecieran señalar que el co-ión tiene algún efecto sobre el proceso de agregación. Esto tiene sentido si consideramos además la naturaleza cosmotrópica o caotrópica del catión proveniente de la sal inorgánica. $\mathrm{Al}$ aumentar la concentración del catión cosmotrópico (magnesio) la agregación debe producirse más fácilmente, puesto que mayor número de moléculas de agua se asociarán al mismo, dejando expuesta la parte hidrofóbica del catión del líquido iónico.

Por tal motivo, se decidió graficar de manera simultánea en la Figura 4 el logaritmo de la CMC como una función lineal de la concentración del co-ión, como sigue:

$$
\log C M C=a^{\prime}+b^{\prime} \cdot \log C_{M^{+}}
$$

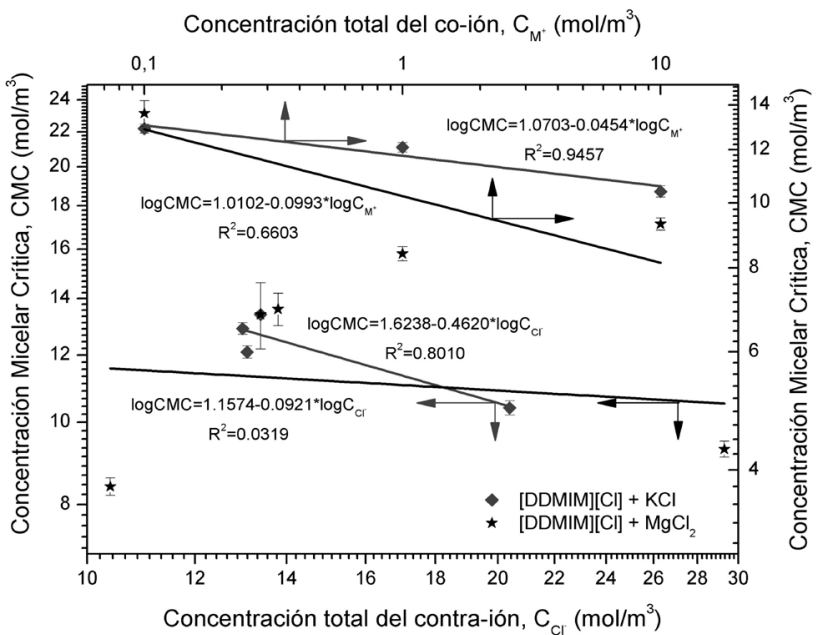

Figura 4. Relación de la CMC con la concentración del co-ión y contra-ión, para el cloruro de 1-dodecil-3-metil-imidazolio

donde $C_{M+}$ es la concentración del co-ión (proveniente exclusivamente de la sal añadida), y $a^{\prime}$ y $b^{\prime}$ son los parámetros a obtener mediante el ajuste.

A pesar de que empleando esta relación los ajustes obtenidos son mucho mejores, sigue manteniéndose la tendencia descrita anteriormente: el ajuste para los datos provenientes de las soluciones acuosas de $\mathrm{KCl}$ resulta ser mucho mejor que el obtenido para las soluciones acuosas de $\mathrm{MgCl}_{2}$.

Esto lleva a pensar que no sólo la naturaleza y la concentración del co-ión tienen un efecto sobre el proceso de agregación, sino que la carga eléctrica asociada al co-ión cumple también un papel preponderante, aspecto mencionado también en un estudio similar llevado a cabo con surfactantes catiónicos del tipo amonio cuaternario. ${ }^{15} \mathrm{La}$ bondad de los ajustes asociados al $\mathrm{KCl}$ se explica al considerar que esta sal es del tipo 1:1 en términos de la carga eléctrica asociada a cada ion, al igual que el líquido iónico; mientras que el $\mathrm{MgCl}_{2}$ es una sal del tipo 2:1.

Por estas razones, pareciera lógico pensar que un modelo apropiado para explicar este fenómeno debiera considerar tanto las concentraciones del contra-ión y del co-ión, así como la carga eléctrica asociada a cada uno de ellos. Un parámetro que combina apropiadamente estas condiciones es la fuerza iónica de la solución (I), definida como:

$$
I=\frac{1}{2} \cdot \sum_{i=1}^{n} C_{i} \cdot z_{i}^{2}
$$

donde $C_{i}$ es la concentración del ion $i$, mientras que $z_{i}$ es la carga eléctrica asociada a dicho ión.

Para ello se empleó el modelo desarrollado por Huang et al. ${ }^{16}$ para explicar el efecto que la adición de sales inorgánicas tienen sobre la formación de micelas en soluciones de lecitina. Estos autores, basándose en un modelo de la energía libre de Gibbs para soluciones acuosas en presencia de sales, llegaron a una expresión del tipo:

$$
\log C M C=a^{\prime \prime}+b^{\prime \prime} \cdot I^{1 / 2}+c^{\prime \prime} \cdot I
$$

donde $a$ ", $b$ " y $c$ " son los parámetros a obtener mediante el ajuste.

En la Figura 5 se observan los ajustes obtenidos para ambas sales, apreciándose que la bondad del ajuste para el $\mathrm{KCl}$ es prácticamente la unidad y que la bondad del ajuste para el $\mathrm{MgCl}_{2}$ mejoró de forma considerable comparado con la obtenida para los modelos representados por las Ecuaciones 5 y 6 . Estos resultados permiten entonces 


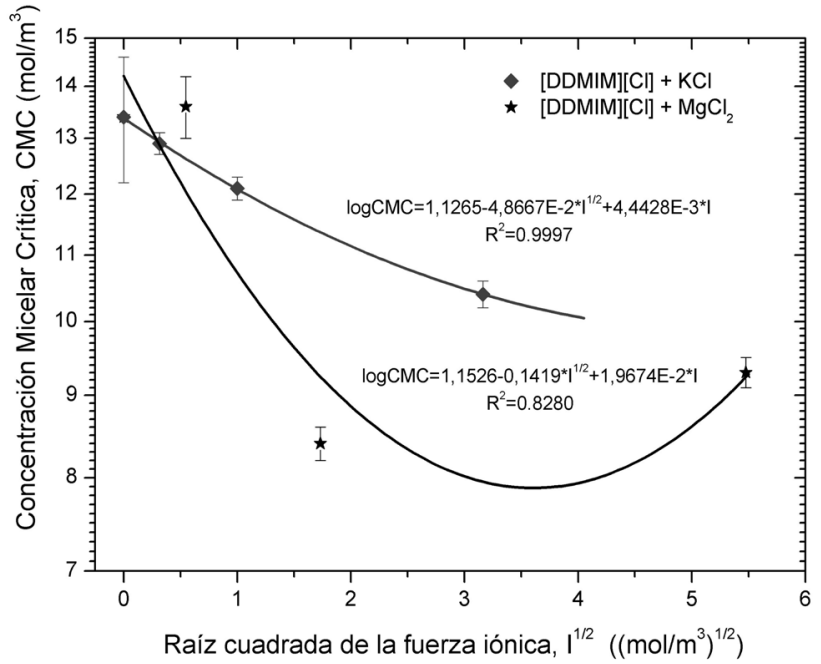

Figura 5. Relación de la CMC con la fuerza iónica de la solución, para el cloruro de 1-dodecil-3-metil-imidazolio

afirmar que la agregación de líquidos iónicos en solución acuosa y en presencia de sales inorgánicas está afectada por los factores desarrollados a lo largo de este estudio.

Una investigación reciente ${ }^{17}$ realizada con un líquido iónico de la familia piridinio muestra que la adición de sales inorgánicas afecta el proceso de micelización al considerar efectos eléctricos, de mezclado intersticial y cosmotrópicos sobre el solvente. Señalan además que tanto el co-ión como el contra-ión juegan un papel importante, pero que en muchos casos el efecto dominante del contra-ión suprime el efecto del co-ión, aunque el co-ión sigue teniendo efecto sobre los parámetros asociados a las micelas formadas.

Finalmente, se deben realizar esfuerzos en ampliar la cantidad de datos experimentales que describan la agregación de líquidos iónicos en presencia de sales inorgánicas, pero teniendo en cuenta la cantidad inmensa de líquidos iónicos que es posible crear mediante combinaciones de diversos aniones y cationes (más de $10^{18}$ según Vagt et al.), ${ }^{18}$ se debe ser muy cuidadoso a la hora de escoger las combinaciones posibles, para reducir la posibilidad de que haya intercambio de iones o se formen nuevos líquidos iónicos.

Respecto a este último punto, Fernández et al. ${ }^{19}$ reportan el caso del $\mathrm{FeCl}_{3}$ con cloruro de 1-octil-3-metilimidazolio, donde el cloruro férrico puede asociarse al anión cloruro del líquido iónico para formar un nuevo anión $\left(\mathrm{FeCl}_{4}^{-}\right)$y, por lo tanto, la CMC determinada es producto de la mezcla de dos líquidos iónicos de naturaleza diferente.

\section{CONCLUSIONES}

A partir del procesamiento de los datos experimentales obtenidos a través de las mediciones de conductividad eléctrica y tensión superficial de trece líquidos iónicos de la familia 1-alquil-3-metilimidazolio en solución acuosa se concluye que a medida que aumenta la longitud de la cadena carbonada se observa una disminución de la CMC. Por el contrario, para el caso de una cadena carbonada corta no se detectó agregación.

El hecho de emplear un anión cada vez más grande reduce la tensión superficial, pero no favorece la formación de agregados.

A medida que se aumenta el tamaño del anión, se reduce la solubilidad del líquido iónico en agua de forma drástica.

La CMC disminuye rápidamente con el incremento del volumen molecular del anión.

$\mathrm{LaCMC}$ obtenida en presencia de $\mathrm{KCl}_{\text {y }} \mathrm{MgCl}_{2}$ es mucho menor a la reportada en ausencia de electrolitos, indicando el efecto de dichas sales sobre la agregación.

La agregación de líquidos iónicos en solución acuosa y en presencia de sales inorgánicas está afectada tanto por la naturaleza y concentración del co-ión como del contra-ión.

\section{MATERIAL SUPLEMENTARIO}

El material disponible en http://quimicanova.sbq.org.br en forma de archivo PDF, con acceso gratuito, contiene información que permite ratificar algunas de las discusiones de los resultados obtenidos en este estudio para líquidos iónicos de la familia 1-alquil-3-metilimidazolio. Las figuras que se presentan son: Dependencia de la CMC con la longitud de la cadena alquílica (Figura 1S); Dependencia de la CMC con el volumen molecular del anión para diversos cationes (Figura 2S); Relación entre el volumen del catión y la longitud de la cadena alquílica (Figura 3S); Efecto de la estructura del catión y del anión sobre la agregación (Figura 4S); y Agregación de [DDMIM] $[\mathrm{Cl}]$ en presencia de: (a) $\mathrm{KCl}$ y (b) $\mathrm{MgCl}_{2}$ (Figura 5S).

\section{AGRADECIMIENTOS}

Al apoyo económico brindado por el Consejo de Desarrollo Científico y Humanístico $(\mathrm{CDCH})$ de la Universidad Central de Venezuela, bajo la figura de los proyectos PI-08-00-6306-2006 y PI-08-00-6307-2006. Así mismo, agradecen al Lic. C. Orbegozo y a la Lic. L. Rodríguez por su apoyo desde MERCK Venezuela para la obtención de las muestras de líquidos iónicos empleadas en esta investigación.

\section{REFERENCIAS}

1. Luczak, J.; Hupka, J.; Thöming, J.; Jungnickel, C.; Colloids Surf., A 2008, 329, 125.

2. Fletcher, K. A.; Pandey, S.; Langmuir 2004, 20, 33.

3. Myers, D.; Surfactant Science and Technology, VCH Publishers: New York, 1988.

4. Baker, R. J.; Acree, W. E.; Tsai, C. C.; QSAR 1984, 3, 10

5. Klein, R. ; von Grünberg, H. H.; Pure Appl. Chem. 2001, 73, 1705.

6. Kalra, A.; Tugcu, N.; Cramer, S. M.; Garde, S.; J. Phys. Chem. B 2001, 105, 6380 .

7. Miskolczy, Z.; Sebök-Nagy, K.; Biczok, L.; Göktürk, S.; Chem. Phys. Lett. 2004, 400, 296.

8. Stewart, J. J. P.; J. Mol. Model. 2007, 13, 1173.

9. Hiemenz, P. C.; Rajagopalan, R.; Principles of Colloid and Surface Chemistry, $3^{\text {rd }}$ ed., Marcel Dekker Inc.: New York, 1997.

10. Forsyth, S. A.; Pringle, J. M.; MacFarlane, D. R.; Aust. J. Chem. 2004, 57, 113.

11. Hunter, R. J.; Foundations of colloid science, Oxford University Press: Oxford, 1989.

12. Blesic, M.; Marques, M. H.; Plechkova, N. V.; Seddon, K. R.; Rebelo, L. P. N.; Lopes, A.; Green Chem. 2007, 9, 481.

13. Zhao, H.; J. Chem. Technol. Biotechnol. 2006, 81, 877.

14. Corrin, M. L.; Harkins, W. D.; J. Am. Chem. Soc. 1947, 69, 683

15. Jiang, N.; Li, P.; Wang, Y.; Wang, J.; Yan, H.; Thomas, R. K.; J. Colloid Interface Sci. 2005, 286, 755.

16. Huang, Y. X.; Tan, R. C.; Li, Y. L.; Yang, Y. Q.; Yu, L.; He, Q. C.; J. Colloid Interface Sci. 2001, 236, 28.

17. Bhat, M. A.; Dar, A. A.; Amin, A.; Rather, G. M.; J. Dispersion Sci. Technol. 2008, 29, 514.

18. Vagt, U.; Emanuel, C.; Chem. Processing 2006, 69, 45.

19. Fernández, J. F.; Waterkamp, D.; Thöming, J.; Desalination 2008, 224, 52. 


\section{EFECTO DEL CATIÓN, DEL ANIÓN Y DEL CO-IÓN SOBRE LA AGREGACIÓN DE LÍQUIDOS IÓNICOS EN SOLUCIÓN ACUOSA}

Nólides Marina Guzmán, José Francisco Fernández*, Monica Parada, Carlos Orbegozo, María Alejandra Rodríguez y Alida Padrón

Escuela de Ingeniería Química, Universidad Central de Venezuela, 1040 Caracas, Venezuela

Jorg Thöming

Zentrum für Umweltforschung und nachhaltige Technologien, Universität Bremen, 28359 Bremen, Deutschland

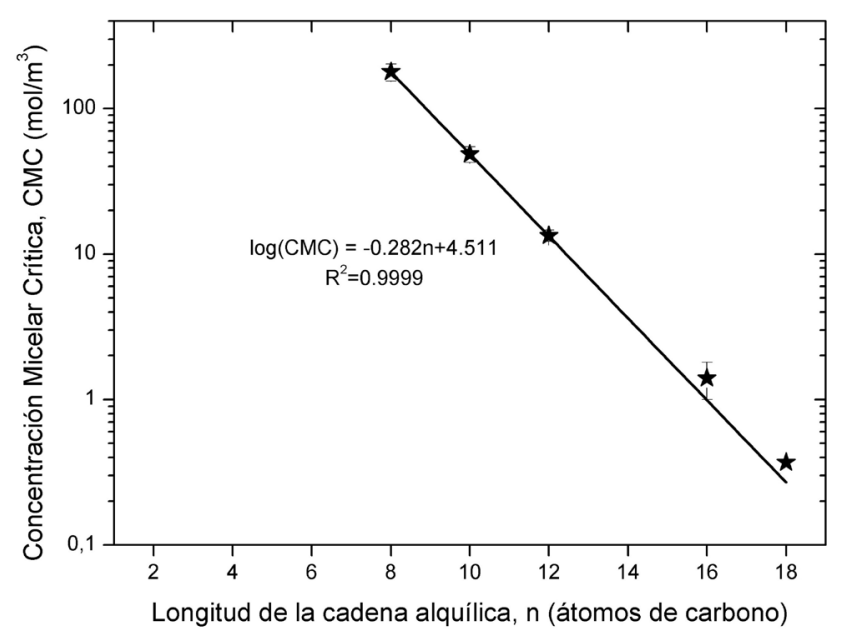

Figura 1S. Dependencia de la CMC con la longitud de la cadena alquílica para líquidos iónicos de la familia 1-alquil-3-metil-imidazolio

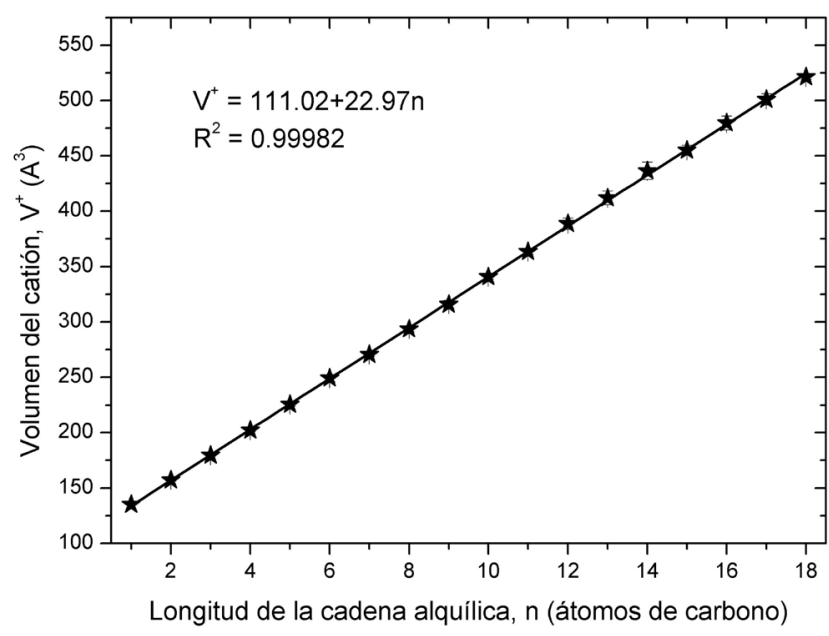

Figura 3S. Relación entre el volumen del catión y la longitud de la cadena alquílica para líquidos iónicos de la familia 1-alquil-3-metil-imidazolio

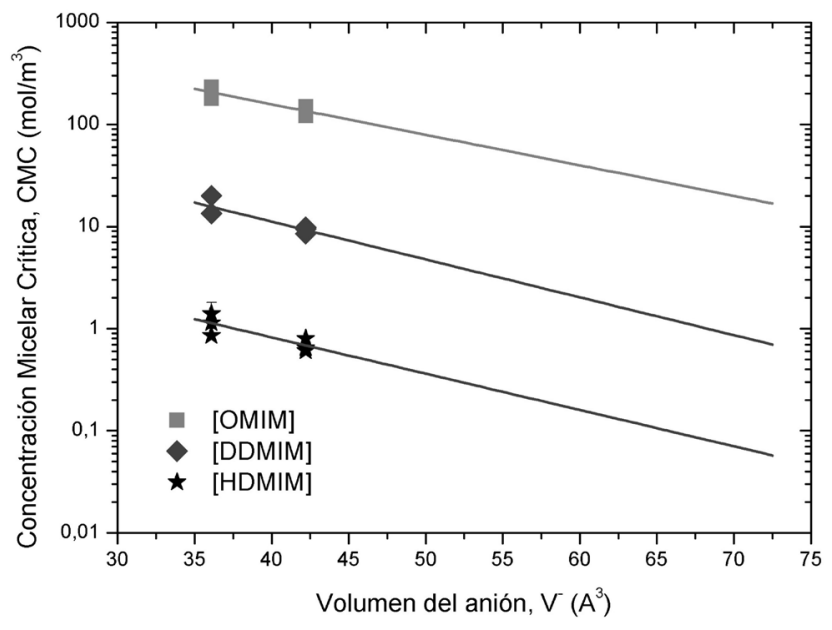

Figura 2S. Dependencia de la CMC con el volumen molecular del anión para diversos cationes de la familia 1-alquil-3-metil-imidazolio

*e-mail: jo_fe@uni-bremen.de 

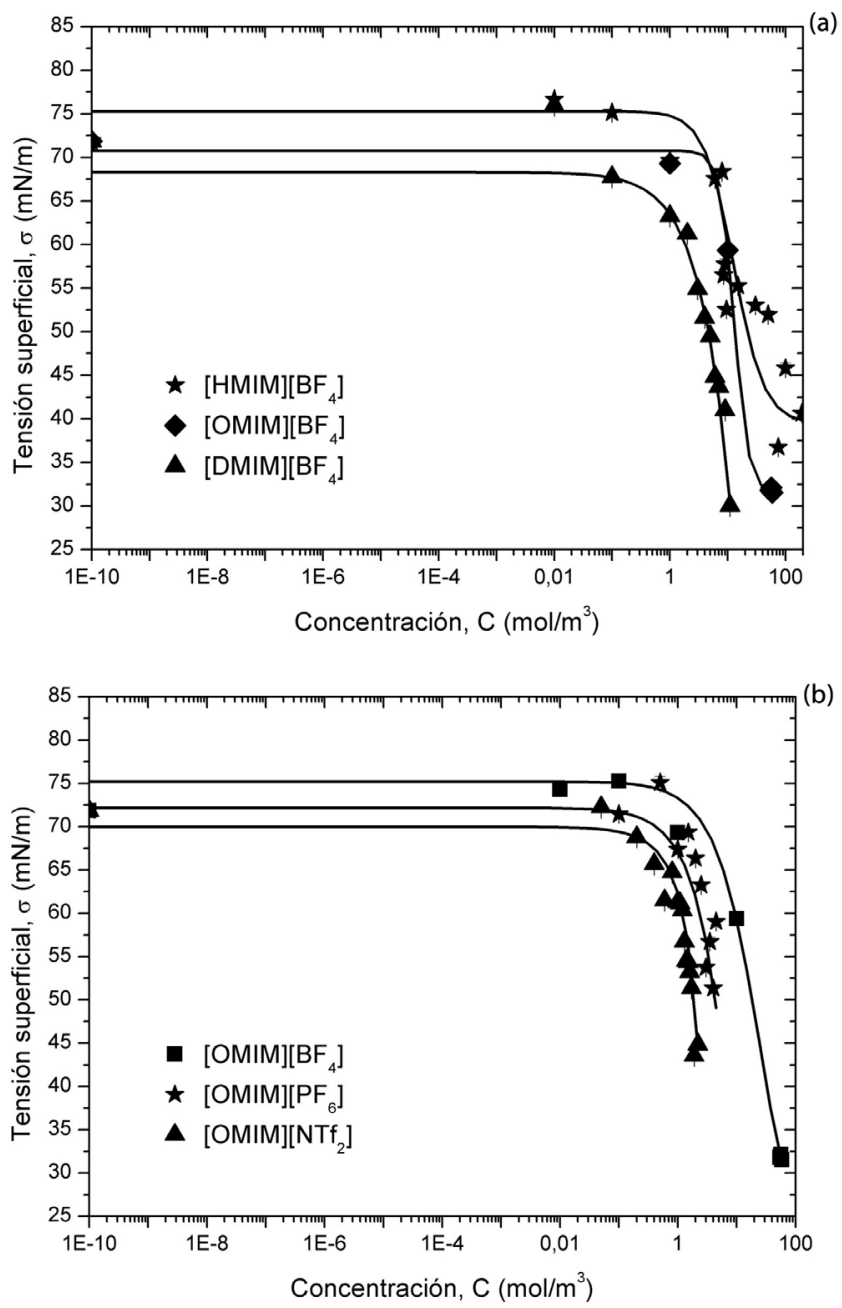

Figura 4S. Efecto de la estructura del (a) catión, (b) anión, sobre la agregación de líquidos iónicos de la familia 1-alquil-3-metil-imidazolio

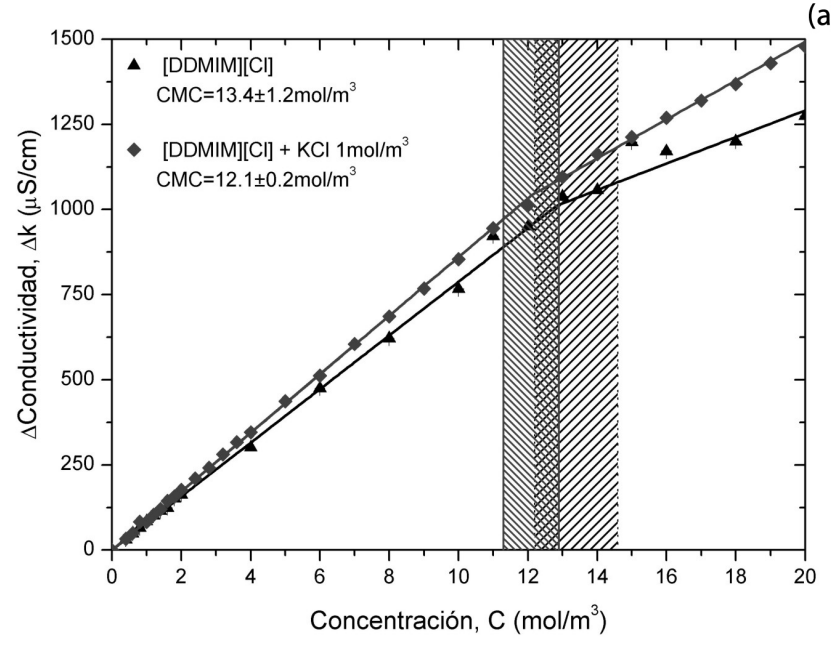

(a)

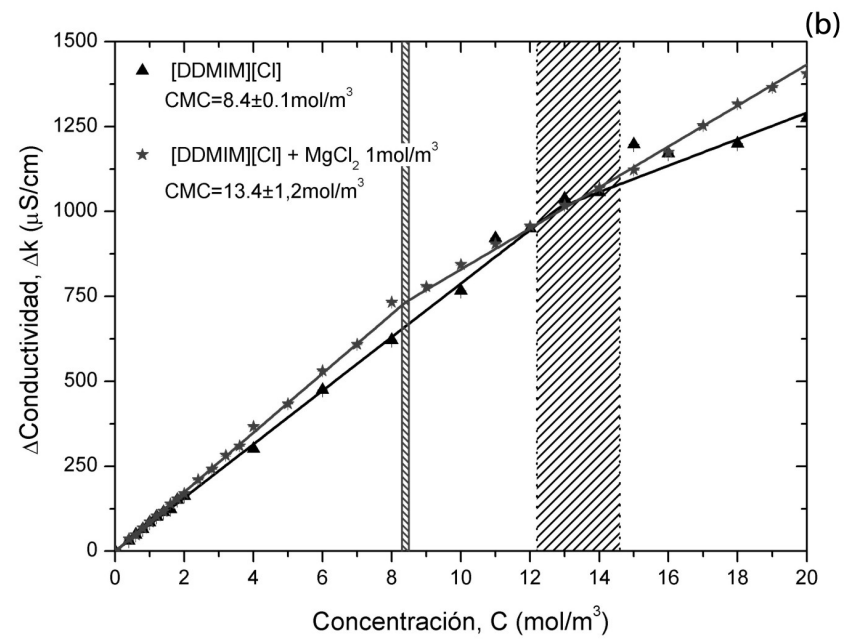

Figura 5S. Agregación de cloruro de 1-dodecil-3-metil-imidazolio en presencia de: (a) $\mathrm{KCl}$ y (b) $\mathrm{MgCl}_{2}$ 